\title{
(C) OPEN ACCESS \\ Human cerebral evolution and the clinical syndrome of amyotrophic lateral sclerosis
}

\author{
Robert D Henderson, ${ }^{1}$ Fleur C Garton, ${ }^{2}$ Matthew C Kiernan, ${ }^{3}$ Martin R Turner, ${ }^{4}$ \\ Andrew Eisen ${ }^{5}$
}

'Department of Neurology, Royal Brisbane and Women's Hospital, Brisbane, Queensland, Australia

${ }^{2}$ Institute for Molecular Bioscience, University of Queensland, Brisbane, Queensland, Australia ${ }^{3}$ Brain \& Mind Centre, University of Sydney, Sydney, New South Wales, Australia

${ }^{4}$ Nuffield Department of Clinical Neurosciences, Oxford University, Oxford, UK ${ }^{5}$ Division of Neurology Department of Medicine, University of British Columbia, Vancouver, British Columbia, Canada

\section{Correspondence to}

Associate Professor Robert D Henderson, Department of Neurology, Royal Brisbane \& Women's Hospital, Herston QLD 4029, Australia; robert. henderson@health.qld.gov.au

Received 10 February 2018 Revised 23 March 2018

Accepted 29 March 2018 Published Online First 17 April 2018

Check for updates

To cite: Henderson RD, Garton FC, Kiernan MC, et al. J Neurol Neurosurg Psychiatry 2019:90:570-575.

\section{INTRODUCTION}

The adult neurodegenerative disorder amyotrophic lateral sclerosis (ALS) is unmistakeable from clinical descriptions now more than a century old. ${ }^{1}$ Neither the site of symptom onset in $\mathrm{ALS}^{2}$ nor pattern of symptom spread ${ }^{3-5}$ is truly random. While the most consistent signs relate to motor system dysfunction, it is recognised that ALS represents an anatomically widespread neocortical disease, ${ }^{6}$ involving significant pathological and genetic overlap with Frontotemporal Dementia (FTD). ${ }^{78}$ Nearly all cases of ALS, and half of FTD, are associated with cytoplasmic, ubiquitinated inclusions of aggregated TDP-43. ${ }^{9}$ The linkage of apparently disparate cortical networks in the ALS-FTD pathological spectrum has been conceptualised as 'what wires together, fires together and dies together', ${ }^{10}$ based on a wider concept that neurodegenerative syndromes are defined by the architecture of large-scale brain networks. ${ }^{11}$ The motor system is perhaps one of the most fundamental to evolution, and the relative rarity of its targeted disintegration within the spectrum of neocortical disorders ${ }^{12}$ encapsulates a fundamental conundrum of understanding selective vulnerability in cerebral neurodegeneration. ${ }^{13}$

Approximately 600000 years ago, Homo sapiens evolved from a common ancestor along with Neanderthal and other Hominid species, with evidence that Homo Sapiens of African origin exhibited the greatest genomic diversity. ${ }^{14-16}$ During this time, efficient cerebral processing for complex functioning evolved. This included versatile vocalisation accompanied by complexity of respiration, language and associated socialisation, increased fractionation of digits and thumb opposability, and upright walking with ability to navigate uneven and tricky surfaces while erect (figure 1). Failure of all of these functions characterises clinical manifestations of ALS. ${ }^{17}$ Paralleling functional advances, the shape and development of the brain have also evolved, ${ }^{18}$ including a diversification of different cell types. To achieve the fast, efficient motor processing required, particular motor output systems developed, best exemplified by the monosynaptic corticomotoneuronal system. Efficient processing was further enhanced by increased cerebral dominance, where laterality could maximise efficiency. We now consider the evolutionary biology of hemispheric specialisation in more depth as it might pertain to selective vulnerability in ALS.

\section{Hemispheric dominance}

Given that there is widespread cerebral dominance in vertebrate brains, and even some invertebrates, it seems reasonable to assume that this must confer favourable selective advantages, ${ }^{19}{ }^{20}$ possibly by increasing neural capacity, within the limitations of a bony skull. ${ }^{21}$ Specialisation within each hemisphere enables the brain to control specific functions such as handedness, gesturing and speech, but also vision and other functions such as sleep. ${ }^{22}$ Humans are uniquely predominantly right-handed, and most right-handed individuals are left hemisphere dominant for language. Typically, 97\% of right-handers demonstrate left-brain preference for language but so too do $60 \%$ of left-handers. ${ }^{23} 24$

Cerebral dominance is limited in non-primates, becoming increasingly evident with primate evolution, and most apparent in humans. ${ }^{23}$ Nevertheless, left-right asymmetries are found in many animal species (action dynamics for aquatic mammals, vocalisation for mice and amphibians). ${ }^{25}$ Humans however have the most consistent population-wide bias towards cerebral laterality, and impaired dominance is associated with a number of disorders (such as autism and perhaps schizophrenia). ${ }^{26-28}$

Left hemispheric preference in primate development possibly occurred as a random event, although evidence favours a link with language dominance, in that language dominance and handedness were considered to have co-evolved in hominid evolution. ${ }^{25} 29$ However, contrary evidence from behavioural and neuroimaging studies challenges this and biological, functional and social factors have also been shown to be determinants of hand dominance. ${ }^{30}$ For example, hand preferences in non-human primates reveal evidence of population-level handedness, but during some tasks, requiring controlled posture and reaching, the majority of prosimians show left-handedness. ${ }^{31}$ In Old and New World monkeys as well as great apes, coordinated bimanual tasks show consistent evidence of population-level handedness. In rhesus monkeys and chimpanzees, there is preferential use of the right hand for gesturing, and this is enhanced when accompanied by a vocalisation. ${ }^{32}$ While other species have varying degrees of dominance, humans have the most consistent population-wide bias toward right upper limb dominance. ${ }^{25}$ This preference in modern man for right-handedness possibly dates to at least 500000 years, ${ }^{33}$ perhaps to a linkage with the use of tools (figure 1).

Two theories have developed to explain specialisation of the left hemisphere for language, 

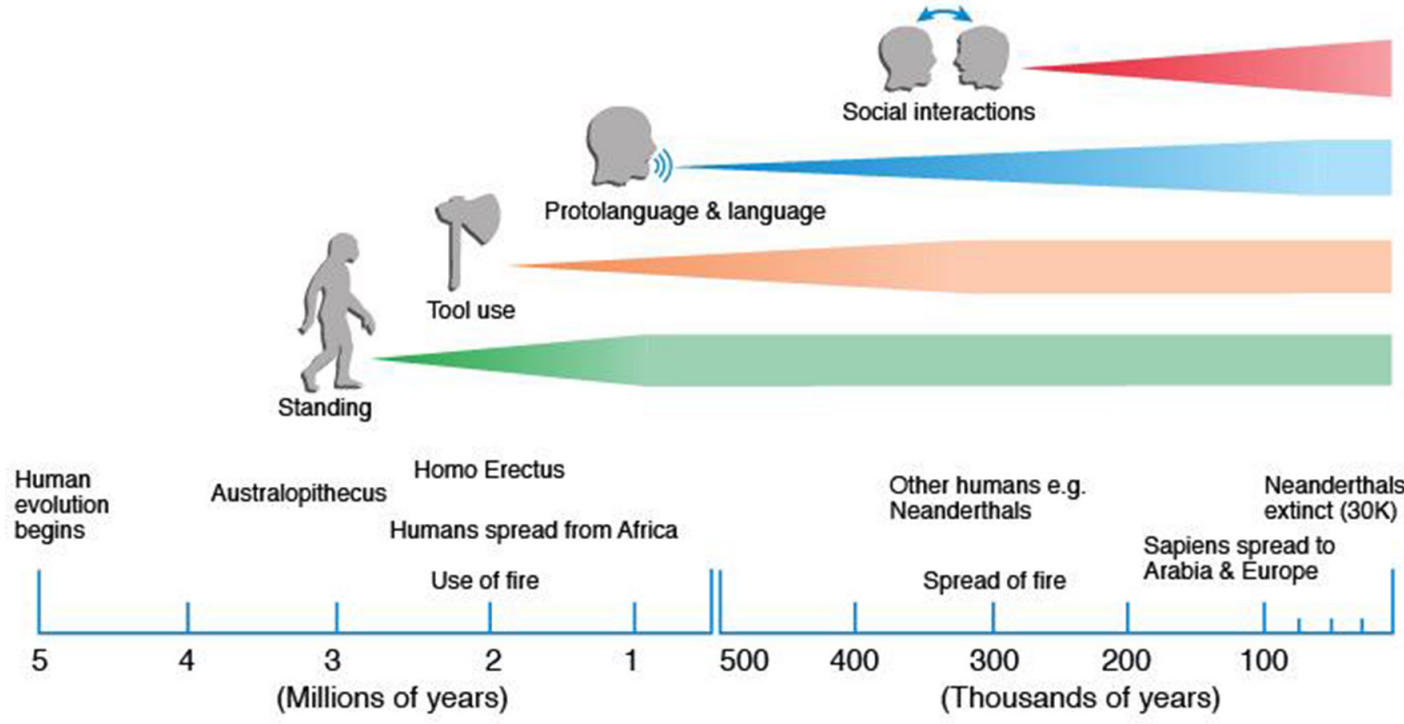

Figure 1 A timeline of human development within the concept that this is very approximate and likely to change with new information which typically causes the lines to move to the left. The definitions of use of tools and social interaction are not defined. Standing and use of tools occurred across different humans (eg, including Neanderthals) but language and social interaction in the last 70000 years is only clear for Homo sapiens.

handedness and other functions. ${ }^{32}$ One advocates that specialisation is primarily genetically determined becoming evident at an early developmental stage, predisposing one hemisphere to 'take the lead'. ${ }^{34}$ An alternative model promotes the corpus callosum as key during prenatal and postnatal development enhancing cross-hemispheric communication. ${ }^{35}$ Neuronal influences are mediated through the fibres of the corpus callosum, which arise from cortical pyramidal cells that are largely glutamatergic, affecting an excitatory role, although they are also known to act through gamma aminobutyric acid-mediated inhibitory neurons. ${ }^{36}$ At a functional level, the corpus callosum enhances processing across the cerebral hemispheres (excitation theory). ${ }^{37}$ Alternatively, its primary function could be transmission of information across cortical fields to suppress one area while the contralateral hemisphere is active. ${ }^{38}$ There is a possible trade-off between relative corpus callosum size and the speed and architecture of the fibres traversing the corpus callosum. ${ }^{32}$

Cerebral dominance can also be considered within global cerebral connectivity concepts where there are favoured resting state networks that facilitate pathways of common use. ${ }^{39} 40$ Nodal signalling and other pathways appear to have a genetic basis, ${ }^{21}$ suggesting that these pathways developed over a long evolutionary period of time, across many animal species, ${ }^{21}$ and ultimately for specific human brain processing functions such as right-hand dominance, language and socialisation. ${ }^{41}$

As the human brain adapted to accommodate more complex motor and social skills, there was a need for fast, efficient cerebral processing within the cerebral cortex and particularly the frontal lobes, which expanded disproportionately to other brain regions. ${ }^{42} 43$ This was accompanied by extensive structural changes, including globularisation, ${ }^{18}$ neural pruning, myelination and synaptogenesis, proceeding from the first year of life through to early teenage years. ${ }^{29} 44$ At the same time, complex neural networks disproportionately evolved over a relatively shorter time span, enabling specific human functions. ${ }^{45}$ Such developments potentially rendered the neocortex, in particular more vulnerable to neurodegeneration. ${ }^{12}$

An illustration of one such possible association of the concepts of lateralised circuits and evolution could be the evolutionary use of fire. Humans obtained an ability to use fire relatively late in evolutionary terms. This required use of tools to 'make it and keep it', with the development of handedness becoming a distinct advantage. Further, social interaction occurred as people gathered for the benefits of fire including warmth, cooking, photoperiodicity and the ability to disperse to colder climates, ${ }^{46}$ accelerating the development of language which was needed as people interacted. All of these cerebral modifications developed over a relatively rapid period with vulnerability of neurons to the effects of ageing.

\section{THE ALS-FTD SYNDROME AS A DISORDER OF VULNERABLE CEREBRAL NETWORKS}

There is increasing appreciation that ALS and FTD are disorders of impaired cerebral network connectivity. ${ }^{47}$ ALS can be considered, at its clinical core, to be a progressive disruption of the complex connection between the corticomotoneuron and targets muscles that implement specific adaptive, complex functions. ${ }^{48}$ Failure of the corticomotoneuronal system preferentially involves the complex adaptive motor skills that are most highly developed in modern humans, such as control of the thumb and index finger, upright walking and speech. ${ }^{17} 49$ The focal nature of ALS is exemplified, by the preferential involvement of the lateral portion of the hand, termed the 'split hand syndrome'. ${ }^{50} 51$ There is greater cortical representation and connectivity of the thenar hand ${ }^{14}$ and current evidence favours a disease mechanism occurring at a cortical level. ${ }^{52-54}$ The focal loss of functionality of the thumb in ALS as a reflection of evolutionary motor system specialisation was enhanced by the recognition that speech (a later evolutionary development) is involved before swallow in ALS, particularly in those with upper motor neuron pathology, ${ }^{55}$ and a preferential involvement of gait, ${ }^{54}$ language ${ }^{56}$ and social awareness across the ALS-FTD spectrum. ${ }^{17}$

Although overt clinical deficit is likely to be a late manifestation of ALS pathology, ${ }^{57}$ once apparent, symptoms inexorably progress in the region where the disease commenced. In some patients, it remains initially restricted to that region for an extended period of time, while in others it appears to have 

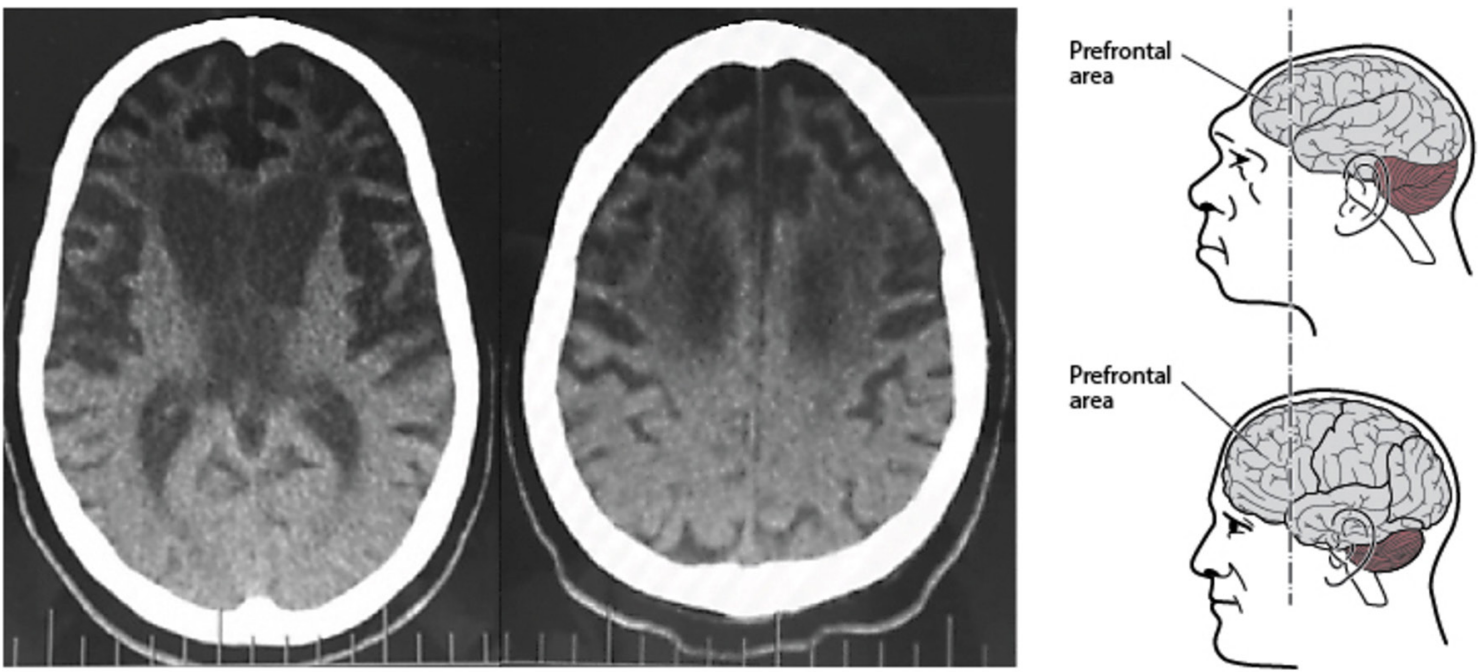

Figure 2 Striking atrophy of frontal lobe regions in a patient with ALS who remained in a persistent vegetative state for 7 years. The clinical presentation was typical of ALS but cognitive studies were not performed. Similarities of the loss of frontal lobe regions with the limited development of these regions in primates and possibly early humans is depicted in the right-hand panel. ALS, amyotrophic lateral sclerosis.

a multifocal and rapid progression. ${ }^{58}$ In ALS, the relatively long preservation of eye movements and sphincter function, both innervated through indirect, polysynaptic cortical pathways, which are in evolutionary terms, old and conserved, as opposed to direct corticomotoneuronal connections which are of recent origin and therefore more vulnerable, would further support evolutionary concepts. ${ }^{48}$

The non-motor cerebral regions may show some of the greatest atrophy when the disease is well established. ${ }^{59}$ As a case in point, an ALS case where the disease process continued while the patient was mechanically ventilated in a persistent vegetative state for 8 years illustrated the striking pathological involvement mainly of the frontal lobe regions (figure 2). Atrophy that occurs in these frontal areas is morphologically opposed to the cortical development that has developed in these regions through evolution from the primate.

\section{Applying lateralisation of cerebral functions to ALS}

In ALS, cerebral dominance in relation to handedness (though not necessarily foot preference) appears to influence clinical presentation ${ }^{2}$ and progression, ${ }^{5}$ so that upper limb onset ALS is more likely to occur in the dominant hand. Spread of weakness beyond the limb of onset also correlated with cerebral dominance, with onset in the non-dominant side more likely to progress to the ipsilateral non-dominant limb in comparison with the onset on the dominant side which spreads to the other contralateral side at that level. ${ }^{558}$ Similar patterns of contiguous spread have been noted in those with lower limb onset. ${ }^{4}$ These suggest a potentially important role for central (upper motor neuron) connections in influencing disease asymmetry. Supporting this theory, neuroimaging has demonstrated disproportionate atrophy of relevant cortical regions in patients with ALS with onset in the dominant hand ${ }^{60}$ (figure 3 ).

Cerebral dominance (a normal evolutionary process) and pathological processes can both be considered as a brain network connectivity modification, with the corpus callosum as an important determinant. ${ }^{32}$ Degeneration of the corpus callosum is a consistent finding in ALS pathology and is most salient within motor-associated fibre regions. ${ }^{61-63}$ Postmortem diffusion imaging has enabled correlation of microstructural imaging changes with the corresponding histological changes of axonal loss, astrocytosis and microglial infiltration, ${ }^{64}$ most prominent in patients carrying a hexanucleotide expansion in C9orf72, the most common hereditary form of ALS and behavioural variant FTD.

Local and regional cortical communication is associated with continuous rhythmic neuronal oscillations, which during movement preparation and execution, modulate at an oscillation of $15-30 \mathrm{~Hz}$ (beta), as measured by magnetoencephalography. Augmented beta desynchronisation in both ipsilateral and contralateral motor cortices has been described in patients with ALS during motor preparation. ${ }^{65}$ Further, in symptomatic carriers of genetic mutations, excess beta desynchronisation was

\section{Controls}
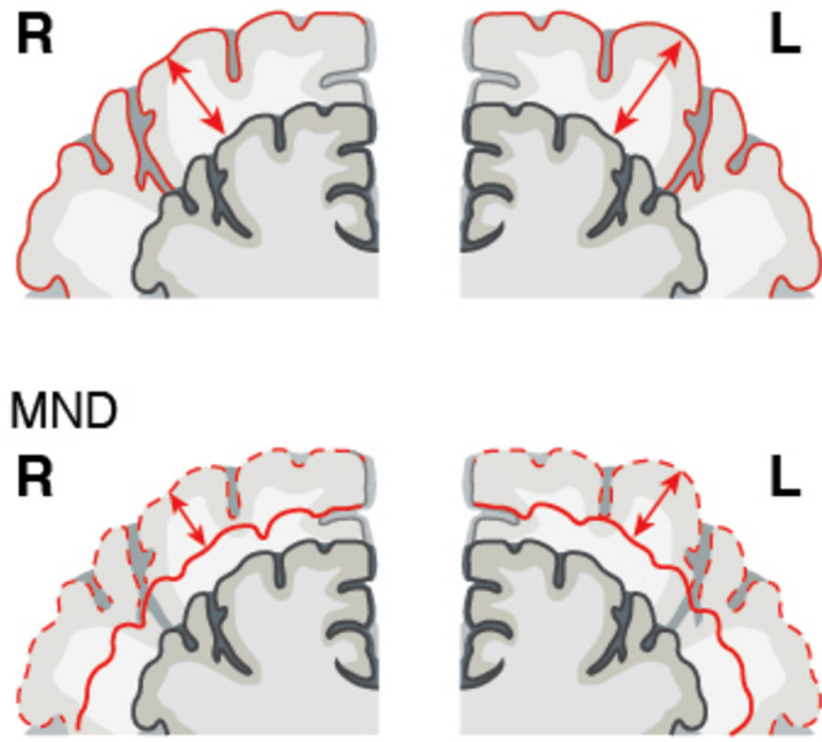

Figure 3 Normally there is cortical asymmetry with a slightly larger and more distinct region in the left hemisphere (top panel). The solid arrow indicates cortical thickness. Loss of this cortical asymmetry occurs in patients with MND (bottom panel) in comparison with controls with preferential involvement (atrophy) of the dominant left hemisphere (arrow). ${ }^{60}$ MND; Motor Neurone Disease. 
recorded with movement execution. Movement completion was followed by a slowed rebound of beta power, postulated to correspond to involvement of interhemispheric fibres of the corpus callosum. Intensified cortical beta desynchronisation followed by delayed rebound is concordant with the broader concept of cortical hyperexcitability in ALS. ${ }^{66}$ Hyperexcitability can be hypothesised to occur to a greater degree in the dominant hemisphere, which has had, over a number of decades, greater functionality.

The evolutionary concepts pertaining to vulnerable cerebral circuits can be practically applied to the unanswered clinical observations in ALS such as the age of onset, gender preference and the lack of occurrence in non-human species or with other diseases. Primates and early humans, who had an expected lifespan of 40-60 years ${ }^{67}$ (ie, a similar to the typical mean age of onset for ALS), evolved lateralised pathways and enhanced cerebral connectivity suited to this lifespan. A clear difference in lateralised circuits is recognised between human genders and can be extended to include primates ${ }^{68}$ and might have relevance for the known gender inequality in ALS. ${ }^{69}$ The lack of occurrence of ALS pathology in non-humans and the relative lack of association of ALS with other organ diseases also become somewhat clearer if the evolution of lateralised cerebral circuits is considered as they pertain to humans.

\section{GENETIC CONSIDERATIONS}

Approximately one quarter of the variation in hand preference can be explained by additive genetic effects, ${ }^{70}$ however, these cannot be assumed to completely reflect cerebral dominance, as the traits are imperfectly related. Brain structure and symmetry correlate with hand preference and speech processing and have higher estimates of variation explained by genetic effects (33\%-53\%). ${ }^{71}$ These correlations imply cerebral dominance is a complex trait, underpinned by polygenic and non-genetic components. The genetic contribution to ALS pathogenesis is also considered complex, with both rare and common variation ${ }^{72-74}$ suggested to be part of a multistep process. ${ }^{75}$ For a carrier of a rare, high-penetrant disease-causing mutation, other factor/s still need to occur or accumulate over time to result in disease.

The presence and frequency of variants in the human genome can be influenced by the demographic histories of populations (ie, migration and natural selection). ${ }^{76}$ Most traits are polygenic and selection can be acting on many trait-associated variants simultaneously each with different effects on related phenotypes. ${ }^{77}$ Measuring genetic conservation in humans may provide a mechanism to understand links between evolution and function. Traditionally, this approach has been relatively limited as the human genome, in evolutionary terms, is relatively young. The amount of time for variants to accumulate for diversity ( $\sim 200000$ years) and the size of the population ( $\sim 10000$ breeding individuals) has resulted in a relatively small number of evolutionary differences between individuals and species. ${ }^{78}$ Put into perspective, with short-read technologies, the absolute sequence difference between chimpanzee and human is estimated to be $\sim 4 \%$ of the genome, which includes coding differences (within the exome) of $\sim 1 \%$ and non-coding changes of $\sim 3 \% .^{79} 80$

Approximately $10 \%$ of ALS cases ${ }^{81}$ have been identified to carry rare coding variants in $\sim 25$ genes. Using available amino acid substitution quotients between human and chimpanzees ${ }^{80}$ and testing for enrichment for loss of function or non-synonymous intolerant genes in an exome population database ${ }^{82}$ do not support a hypothesis that the current known ALS genes have been under any positive selection pressure within or across species. Common single-nucleotide polymorphisms are also estimated to have a role in ALS pathogenesis, ${ }^{7273}$ and while those identified explain $<0.5 \%$ of the heritability, it is hypothesised that associated alleles produce a sub-threshold abnormality that in turn can be inherited. ${ }^{83}$ This suggests a model of 'ancestral neutrality'.

Interestingly, common genetic variants associated with schizophrenia have found to have a higher propensity to be in genomic regions that diverge from their Neanderthal counterparts, than expected by chance. ${ }^{84}$ Such findings suggest that variants are associated with becoming human. ${ }^{85}$ If so, it could be hypothesised that other cerebral diseases developed as a result of genetic specialisation for functions specific to humans ${ }^{86}$ and would have a higher frequency of occurring together. ${ }^{87}$ An alternative theory to 'ancestral neutrality', which has been proposed for variants associated with psychiatric and developmental disorders such as schizophrenia, ${ }^{84} 85$ is that there is both a negative and positive fitness effect that may explain why certain disease variants have been maintained in the population (ie, a balanced selection model). For common variants associated with a disease, evidence of a founder effect may indicate a positive effect that maintains the variant in the population. Multiethnic ALS population studies demonstrate relatively few founder effects (single ancestor) and the majority of ALS variants are rare and arise independently in a common set of known genes (ie, SOD1). ${ }^{88} 89$ An exception to this is the C9orf72 expansion, ${ }^{9091}$ which does suggest a one-off expansion originating in Northern Europe $\sim 6300$ years ago. ${ }^{92}$ A balanced-selection model may suggest that this variant has not decreased in frequency due to a positive selection effect on cerebral connectivity. This is not dissimilar to hypotheses of "creative cognition' whereby human evolution may have favoured this trait, intriguingly however it also may present genetic vulnerability for neurological and psychiatric disorders. ${ }^{93}$

The cellular processes that maintain the normal physiology of the neuronal system throughout life are currently being elucidated. Common genes identified in ALS and FTD function in a number of common molecular pathways involving RNA metabolism and homeostasis. ${ }^{94}{ }^{95}$ In particular, the cellular accumulation of the DNA/RNA-binding protein TDP-43 (encoded by TARDBP) found in $90 \%$ of ALS cases highlights the importance of DNA/RNA homeostasis in neurons. Following DNA transcription, RNA molecules within a cell are bound by distinct sets of RNA-binding proteins that have the task of regulating the correct processing, transport, stability and function/translation up to its final degradation. Increasing age is the main risk factor for accumulation of protein aggregates as seen in neurodegenerative diseases with different protein signalling pathways affected depending on the unfolded protein response. As human lifespan has increased through evolution, there is a theory that cerebral protein aggregation has acted as a constraint on increasing age. ${ }^{96}$

\section{Concluding remarks}

We propose that ALS may be a disease that preferentially affects cerebral structures and pathways that have evolved recently in human evolution. Specifically, it is proposed that ALS-FTD represents a syndrome preferentially involving regions of the brain under relatively recent evolutionary change, whose complexity possibly renders it more vulnerable to insults or stressors that accumulate with ageing. The connectivity pathways serving rapid cerebral processing are sadly the most vulnerable to changes with increasing age. 
Neurodegenerative syndromes have a complex polygenic basis resulting in impairment of neocortical network connectivity. Some profound psychiatric disorders of brain connectivity become clinically overt in much younger adults, but have a greater than expected incidence in those with ALS and their relatives, ${ }^{9798}$ suggesting that neurodevelopment may have important implications for understanding neurodegeneration.

Motor system neurodegeneration, previously neglected, can be applied to the increasing literature relating to evolution and cerebral connectivity that has so far been dominated by language and wider cognitive processing considerations. Such concepts, while they are necessarily speculative, support the classification of ALS among the cerebral neurodegenerative rather than peripheral nerve disorders.

Correction notice Since this article was first published online there have been some text changes in the second paragraph of the introduction.

Acknowledgements Naomi Wray, Matthew Devine and Roger Lemon provided valuable commentary during the evolution of the manuscript.

Contributors RH and AE: study concept and design. All authors: drafting of the manuscript. $\mathrm{RH}, \mathrm{AE}, \mathrm{MK}$ and $\mathrm{MT}$ : critical revision of the manuscript for important intellectual content. RH, FG and MK: administrative, technical or material support.

Funding The authors have not declared a specific grant for this research from any funding agency in the public, commercial or not-for-profit sectors.

Competing interests None declared.

Patient consent Not required.

Provenance and peer review Commissioned; externally peer reviewed.

Open access This is an open access article distributed in accordance with the Creative Commons Attribution Non Commercial (CC BY-NC 4.0) license, which permits others to distribute, remix, adapt, build upon this work non-commercially, and license their derivative works on different terms, provided the original work is properly cited and the use is non-commercial. See: http://creativecommons.org/ licenses/by-nc/4.0/

(c) Article author(s) (or their employer(s) unless otherwise stated in the text of the article) 2019. All rights reserved. No commercial use is permitted unless otherwise expressly granted.

\section{REFERENCES}

1 Mott FW. A case of amyotrophic lateral sclerosis with degeneration of the motor path from the cortex to the periphery. Brain 1895;18:21-36.

2 Turner MR, Wicks P, Brownstein CA, et al. Concordance between site of onset and limb dominance in amyotrophic lateral sclerosis. Journal of Neurology, Neurosurgery \& Psychiatry 2011:82:853-4.

3 Ravits J, Paul P, Jorg C. Focality of upper and lower motor neuron degeneration at the clinical onset of ALS. Neurology 2007;68:1571-5.

4 Turner MR, Brockington A, Scaber J, et al. Pattern of spread and prognosis in lower limb-onset ALS. Amyotrophic lateral sclerosis: official publication of the World. Federation of Neurology Research Group on Motor Neuron Diseases 2010;11:369-73.

5 Devine MS, Kiernan MC, Heggie S, et al. Study of motor asymmetry in ALS indicates an effect of limb dominance on onset and spread of weakness, and an important role for upper motor neurons. Amyotroph Lateral Scler Frontotemporal Degener 2014:15:481-7.

6 Eisen A, Braak H, Del Tredici K, et al. Cortical influences drive amyotrophic lateral sclerosis. J Neurol Neurosurg Psychiatry 2017:88:917-24.

7 Turner MR, Swash M. The expanding syndrome of amyotrophic lateral sclerosis: a clinical and molecular odyssey. Journal of Neurology, Neurosurgery \& Psychiatry 2015:86:667-73.

8 Burrell JR, Halliday GM, Kril JJ, et al. The frontotemporal dementia-motor neuron disease continuum. The Lancet 2016:388:919-31.

9 Neumann M, Sampathu DM, Kwong LK, et al. Ubiquitinated TDP-43 in frontotemporal lobar degeneration and amyotrophic lateral sclerosis. Science 2006;314:130-3.

10 Bak TH, Chandran S. What wires together dies together: Verbs, actions and neurodegeneration in motor neuron disease. Cortex 2012;48:936-44.

11 Seeley WW, Crawford RK, Zhou J, et al. Neurodegenerative diseases target large-scale human brain networks. Neuron 2009;62:42-52.

12 Eisen A, Turner MR. Does variation in neurodegenerative disease susceptibility and phenotype reflect cerebral differences at the network level? Amyotroph Lateral Scler Frontotemporal Degener 2013;14:487-93.
13 Mattsson N, Schott JM, Hardy J, et al. Selective vulnerability in neurodegeneration: insights from clinical variants of Alzheimer's disease. Journal of Neurology, Neurosurgery \& Psychiatry 2016;87:1000-4.

14 Weber M, Eisen A, Stewart H, et al. The split hand in ALS has a cortical basis. I Neurol Sci 2000;180:66-70.

15 Posth C, Wißing C, Kitagawa K, et al. Deeply divergent archaic mitochondrial genome provides lower time boundary for African gene flow into Neanderthals. Nat Commun 2017:8:16046.

16 Nielsen R, Akey JM, Jakobsson M, et al. Tracing the peopling of the world through genomics. Nature 2017:541:302-10.

17 Eisen A, Turner MR, Lemon R. Tools and talk: an evolutionary perspective on the functional deficits associated with amyotrophic lateral sclerosis. Muscle Nerve 2014:49:469-77.

18 Neubauer S, Hublin JJJ, Gunz P. The evolution of modern human brain shape. Sci Adv 2018:4 eaa05961.

19 Rogers LJ AR. Comparative vertebrate lateralization. 2002.

20 Gutwinski S, Loscher A, Mahler L, et al. Understanding left-handedness. Dtsch Arztebl Int 2011;108:849-53.

21 Güntürkün 0, Ocklenburg S. Ontogenesis of lateralization. Neuron 2017;94:249-63.

22 Mascetti, Gian Gastone GG, Mascetti GG. Unihemispheric sleep and asymmetrical sleep: behavioral, neurophysiological, and functional perspectives. Nat Sci Sleep 2016:8:221-38

23 Geschwind N. The organization of language and the brain. Science 1970;170:940-4.

24 Geschwind DH, Miller BL, DeCarli C, et al. Heritability of lobar brain volumes in twins supports genetic models of cerebral laterality and handedness. Proceedings of the National Academy of Sciences 2002:99:3176-81.

25 Fitch WT, Braccini SN. Primate laterality and the biology and evolution of human handedness: a review and synthesis. Ann N Y Acad Sci 2013:1288:70-85.

26 Ribolsi M, Daskalakis ZJ, Siracusano A, et al. Abnormal asymmetry of brain connectivity in Schizophrenia. Front Hum Neurosci 2014;8.

27 Rysstad AL, Pedersen AV. Brief Report: Non-right-Handedness within the autism spectrum disorder. J Autism Dev Disord 2016:46:1110-7.

28 Deep-Soboslay A, Hyde TM, Callicott JP, et al. Handedness, heritability, neurocognition and brain asymmetry in schizophrenia. Brain 2010;133:3113-22.

29 Irurtzun A. The 'Globularization Hypothesis' of the language-ready brain as a developmental frame for prosodic bootstrapping theories of language acquisition. Front Psychol 1817;2015:6.

30 Halpern ME, et al. Lateralization of the vertebrate brain: taking the side of model systems. Journal of Neuroscience 2005:25:10351-7.

31 Hopkins WD, Bennett AJ, Bales SL, et al. Behavioral laterality in captive bonobos (Pan paniscus). J Comp Psychol 1993;107:403-10.

32 Hopkins WD, Misiura M, Pope SM, et al. Behavioral and brain asymmetries in primates: a preliminary evaluation of two evolutionary hypotheses. Ann N Y Acad Sci 2015;1359:65-83.

33 Frayer DW, Lozano M, Bermúdez de Castro JM, et al. More than 500,000 years of right-handedness in Europe. Laterality 2012:17:51-69.

34 Bishop DVM. Cerebral asymmetry and language development: cause, correlate, or consequence? Science 2013:340:12.

35 Jeeves MA, Temple CM. A further study of language function in callosal agenesis. Brain Lang 1987:32:325-35.

36 Palmer LM, Schulz JM, Murphy SC, et al. The cellular basis of GABAB-mediated interhemispheric inhibition. Science 2012:335:989-93.

37 Gazzaniga MS. Cerebral specialization and interhemispheric communication: does the corpus callosum enable the human condition? Brain 2000;123:1293-326.

38 Cook ND. Callosal inhibition: the key to the brain code. Behav Sci 1984;29:98-110.

39 van den Heuvel MP, Mandl RCW, Kahn RS, et al. Functionally linked resting-state networks reflect the underlying structural connectivity architecture of the human brain. Hum Brain Mapp 2009;30:3127-41.

40 Pool E-M, Rehme AK, Eickhoff SB, et al. Functional resting-state connectivity of the human motor network: Differences between right- and left-handers. Neuroimage 2015:109:298-306

41 Corballis MC. The evolution of lateralized brain circuits. Front Psychol 2017;8:1021.

42 Noonan JP. Neanderthal genomics and the evolution of modern humans. Genome Res 2010:20:547-53.

43 Geschwind DH, Rakic P. Cortical evolution: judge the brain by its cover. Neuron 2013:80:633-47.

44 Benes FM. Myelination of cortical-hippocampal relays during late adolescence. Schizophr Bull 1989;15:585-93.

45 Buckner RL, Krienen FM. The evolution of distributed association networks in the human brain. Trends Cogn Sci 2013:17:648-65.

46 Attwell L, Kovarovic K, Kendal J. Fire in the plio-pleistocene: the functions of hominin fire use, and the mechanistic, developmental and evolutionary consequences. $J$ Anthropol Sci 2015;93:1-20

47 Ahmed RM, Devenney EM, Irish M, et al. Neuronal network disintegration: common pathways linking neurodegenerative diseases. Journal of Neurology, Neurosurgery \& Psychiatry 2016;87:1234-41.

48 Eisen A, Kim S, Pant B, et al. ALS): a phylogenetic disease of the corticomotoneuron? Muscle \& nerve 1992;15:219-24. 
49 Devine MS, Farrell A, Woodhouse $H$, et al. A developmental perspective on bulbar involvement in amyotrophic lateral sclerosis. Amyotrophic Lateral Sclerosis and Frontotemporal Degeneration 2013;14(7-8):638-9.

50 Eisen A, Kuwabara S. The split hand syndrome in amyotrophic lateral sclerosis. Journal of Neurology, Neurosurgery \& Psychiatry 2012;83:399-403.

51 Kiernan MC, Turner MR. Lou Gehrig and the ALS split hand. Neurology 2015;85:1995.

52 Bae JS, Menon P, Mioshi E, et al. Cortical hyperexcitability and the split-hand plus phenomenon: Pathophysiological insights in ALS. Amyotrophic Lateral Sclerosis and Frontotemporal Degeneration 2014;15(3-4):250-6.

53 Menon P, Kiernan MC, Vucic S. Cortical hyperexcitability precedes lower motor neuron dysfunction in ALS. Clinical Neurophysiology 2015;126:803-9.

54 Eisen A, Braak H, Del Tredici K, et al. Cortical influences drive amyotrophic lateral sclerosis. Journal of Neurology, Neurosurgery \& Psychiatry 2017;88:917-24.

55 Wakerley BR, Warburton K, Plaha P, et al. Progressive dysphagia without dysarthria. Pract Neurol 2013.

56 Eisen A, Lemon R, Kiernan MC, et al. Does dysfunction of the mirror neuron system contribute to symptoms in amyotrophic lateral sclerosis? Clinical Neurophysiology 2015;126:1288-94.

57 Eisen A, Kiernan M, Mitsumoto H, et al. Amyotrophic lateral sclerosis: a long preclinical period? Journal of Neurology, Neurosurgery \& Psychiatry 2014;85:1232-8.

58 Ravits JM, La Spada AR. ALS motor phenotype heterogeneity, focality, and spread: Deconstructing motor neuron degeneration. Neurology 2009;73:805-11.

59 Menke RAL, Körner S, Filippini N, et al. Widespread grey matter pathology dominates the longitudinal cerebral MRI and clinical landscape of amyotrophic lateral sclerosis. Brain 2014;137(9):2546-55.

60 Devine MS, Pannek K, Coulthard A, et al. Exposing asymmetric gray matter vulnerability in amyotrophic lateral sclerosis. Neuroimage 2015;7:782-7.

61 Probst M. Zur Kenntnis der amyotrophischen Lateralsklerose. S-B Akad Wiss Wien 1903; 112:683-824.

62 Filippini N, Douaud G, Mackay CE, et al. Corpus callosum involvement is a consistent feature of amyotrophic lateral sclerosis. Neurology 2010;75:1645-52.

63 Turner MR, Menke RAL. The tough body at the epicentre of amyotrophic lateral sclerosis. Journal of Neurology, Neurosurgery \& Psychiatry 2017;88:369.2-70.

64 Cardenas AM, Sarlls JE, Kwan JY, et al. Pathology of callosal damage in ALS: an exvivo, 7 T diffusion tensor MRI study. Neuroimage 2017:15:200-8.

65 Proudfoot M, Rohenkohl G, Quinn A, et al. Altered cortical beta-band oscillations reflect motor system degeneration in amyotrophic lateral sclerosis. Hum Brain Mapp 2017;38:237-54.

66 Vucic S, Ziemann U, Eisen A, et al. Transcranial magnetic stimulation and amyotrophic lateral sclerosis: pathophysiological insights. Journal of Neurology, Neurosurgery \& Psychiatry 2013:84:1161-70.

67 Robson SL, Wood B. Hominin life history: reconstruction and evolution. J Anat 2008;212:394-425.

68 Corp N, Byrne RW. Sex difference in chimpanzee handedness. Am J Phys Anthropol 2004; 123:62-8.

69 McCombe PA, Henderson RD. Effects of gender in amyotrophic lateral sclerosis. Gend Med 2010;7:557-70

70 Medland SE, Duffy DL, Wright MJ, et al. Genetic influences on handedness: Data from 25,732 Australian and Dutch twin families. Neuropsychologia 2009;47:330-7.

71 Roshchupkin GV, Gutman BA, Vernooij MW, et al. Heritability of the shape of subcortical brain structures in the general population. Nat Commun 2016;7:13738.

72 van Rheenen W, Shatunov A, Dekker AM, et al. Genome-wide association analyses identify new risk variants and the genetic architecture of amyotrophic lateral sclerosis. Nat Genet 2016;48:1043-8.

73 Benyamin B, He J, Zhao Q, et al. Cross-ethnic meta-analysis identifies association of the GPX3-TNIP1 locus with amyotrophic lateral sclerosis. Nat Commun 2017;8.
74 Garton FC, Benyamin B, Zhao Q, et al. Whole exome sequencing and DNA methylation analysis in a clinical amyotrophic lateral sclerosis cohort. Mol Genet Genomic Med 2017:5:418-28.

75 Al-Chalabi A, Calvo A, Chio A, et al. Analysis of amyotrophic lateral sclerosis as a multistep process: a population-based modelling study. Lancet Neurol 2014;13:1108-13.

76 Fisher R A. The genetical theory of natural selection. Oxford: University Press, 1930:1-308.

77 Pritchard JK, Pickrell JK, Coop G. The genetics of human adaptation: hard sweeps, soft sweeps, and polygenic adaptation. Current Biology 2010;20:R208-15.

78 Jorde LB, Wooding SP. Genetic variation, classification and 'race'. Nat Genet 2004

79 Mikkelsen TS, Hillier LW, Eichler EE, et al. Initial sequence of the chimpanzee genome and comparison with the human genome. Nature 2005;437:69.

80 Varki A, Altheide TK. Comparing the human and chimpanzee genomes: searching for needles in a haystack. Genome Res 2005;15:1746-58.

81 Renton AE, Chiò A, Traynor BJ. State of play in amyotrophic lateral sclerosis genetics. Nat Neurosci 2014;17:17-23.

82 Dolzhenko E, van Vugt J, Shaw RJ, et al. Detection of long repeat expansions from PCR-free whole-genome sequence data. Genome Res 2017;27:093831.

83 Keller MC, Miller G. Resolving the paradox of common, harmful, heritable mental disorders: which evolutionary genetic models work best? Behav Brain Sci 2006;29:385-404; discussion 405-52-404.

84 Srinivasan S, Bettella F, Mattingsdal M, et al. Genetic markers of human evolution are enriched in Schizophrenia. Biol Psychiatry 2016:80:284-92.

85 Polimanti R, Gelernter J. Widespread signatures of positive selection in common risk alleles associated to autism spectrum disorder. PLoS Genet 2017; 13:e1006618.

86 Ogawa LM, Vallender EJ. Evolutionary conservation in genes underlying human psychiatric disorders. Front Hum Neurosci 2014;8.

87 McLaughlin RL, Schijven D, van Rheenen W, et al. Genetic correlation between amyotrophic lateral sclerosis and schizophrenia. Nat Commun 2017;8:14774

88 Saeed M, Yang Y, Deng H-X, et al. Age and founder effect of SOD1 A4V mutation causing ALS. Neurology 2009;72:1634-9.

89 Veldink JH. ALS genetic epidemiology 'How simplex is the genetic epidemiology of ALS?'. Journal of Neurology, Neurosurgery \& Psychiatry 2017:88:537.

90 DeJesus-Hernandez M, Mackenzie IR, Boeve BF, et al. Expanded GGGGCC Hexanucleotide repeat in noncoding region of C9ORF72 Causes chromosome 9p-linked FTD and ALS. Neuron 2011;72:245-56.

91 Renton AE, Majounie E, Waite A, et al. A Hexanucleotide repeat expansion in c9orf72 is the cause of chromosome 9P21-linked ALS-FTD. Neuron 2011;72:257-68.

92 Smith BN, Newhouse S, Shatunov A, et al. The C90RF72 expansion mutation is a common cause of ALS+/-FTD in Europe and has a single founder. European Journal of Human Genetics 2013;21:102-8.

93 Power RA, Steinberg S, Bjornsdottir G, et al. Polygenic risk scores for schizophrenia and bipolar disorder predict creativity. Nat Neurosci 2015;18:953-5.

94 Weishaupt JH, Hyman T, Dikic I. Common molecular pathways in Amyotrophic latera sclerosis and frontotemporal dementia. Trends Mol Med 2016;22:769-83.

95 Kumar V Hasan GM, Hassan MI. Unraveling the role of RNA mediated toxicity of C9orf72 repeats in C9-FTD/ALS. Front Neurosci 2017;11:711.

96 Villar-Pique A, Ventura S. Protein aggregation acts as a strong constraint during evolution. Marseille, France: Springer 2011:103-23.

97 O'Brien M, Burke T, Heverin M, et al. Clustering of Neuropsychiatric Disease in firstdegree and second-degree relatives of patients with amyotrophic lateral sclerosis. JAMA Neurol 2017;74:1425-30.

98 Turner MR, Goldacre R, Talbot K, et al. Psychiatric disorders prior to amyotrophic lateral sclerosis. Ann Neurol 2016;80:935-8. 